\title{
Baseline retinal thickness measurements with a novel integrated imaging system (concurrent optical coherence tomography and fundus photography) positively correlates with spectralis optical coherence tomography
}

\author{
Moisés Enghelberg, Suzie Gasparian, Kakarla V. Chalam^ \\ Department of Ophthalmology, Loma Linda University School of Medicine, Loma Linda, CA, USA \\ Contributions: (I) Conception and design: KV Chalam; (II) Administrative support: KV Chalam; (III) Provision of study materials or patients: All \\ authors; (IV) Collection and assembly of data: S Gasparian, M Enghelberg; (V) Data analysis and interpretation: All authors; (VI) Manuscript writing: \\ All authors; (VII) Final approval of manuscript: All authors.
}

Correspondence to: Kakarla V. Chalam, MD, PhD, MBA, FACS. Professor and Director of Retina, LLU School of Medicine, Department of Ophthalmology, 11370 Anderson Street, Suite 2025, Loma Linda, CA 92354, USA. Email: kvchalam@llu.edu.

Background: Traditionally fundus photographs and optical coherence tomography (OCT) are obtained
separately during evaluation of retinal pathology. We describe a novel integrated imaging system (Monaco,
Optos) that records both OCT as well as fundus photography concurrently. The present study aims to
measure retinal thickness and compare it to OCT obtained with traditional spectral domain OCT in subjects
without known retinal disease to establish normative data for clinical use.
Methods: In this cross sectional study, fundus photographs and OCT was obtained concurrently in 34 eyes
in healthy patients without any known retinal disease with integrated imaging system. OCT with spectralis
was also obtained at the same visit for comparison. All subjects underwent a complete ophthalmologic exam
to ensure the absence of ocular pathology. OCT was performed by the same operator. Central subfield
thickness (CST), central point thickness (CPT), and retinal thickness in nine central subfields were measured
with both 1 instruments. Fundus photographs were obtained. Students $t$-test was used to determine statistical
significance. Results: The mean CST as measured with MIIS-SD OCT and Spectralis OCT was $300.53 \pm 22.81 \mu \mathrm{m}$ and $265.18 \pm 17.33 \mu \mathrm{m}(\mathrm{P}<0.001)$ respectively. The Pearson's correlation coefficient, $\mathrm{r}$ value was 0.5285 , $\mathrm{P}<0.0013$. The mean CPT as measured with MIIS-SD OCT and Spectralis OCT was $268.55 \pm 20.70$ and $230.67 \pm 17.75 \mu \mathrm{m}(\mathrm{P}<0.001)$ respectively. The $\mathrm{r}$-value was $0.5697, \mathrm{P}<0.0004$. The mean difference between retinal thicknesses was $44.88 \mu \mathrm{m}$ (range, 21-91 $\mu \mathrm{m}$ ) in the eight ETDRS subfields, with $\mathrm{r}$ value $0.53, \mathrm{P}<0.05$, ranging from 0.51 to 0.60 . Concurrently obtained ultrawide angle fundus photographs revealed $\left(200^{\circ}\right)$ clear media, normal disc, normal vasculature and normal periphery in all patients with excellent resolution.

Conclusions: Retinal thickness measurements strongly correlated with those obtained by Spectralis. An increased measurement in thickness of $35.35 \mu \mathrm{m}$ was noted in the central fovea. In addition, wideangle fundus photography was successfully obtained in all subjects. Integrated system provides quality fundus photographs as well as OCT, obviates the need for two separate instruments and likely improves the clinic flow.

Keywords: Optical coherence tomography (OCT); macular edema; fundus photography; retinal diseases; age related macular degeneration; diabetic retinopathy

\footnotetext{
$\wedge$ ORCID: 0000-0002-0004-9416.
} 
Submitted Nov 10, 2020. Accepted for publication Jun 17, 2021.

doi: 10.21037 /qims-20-1252

View this article at: https://dx.doi.org/10.21037/qims-20-1252

\section{Introduction}

In the last decade, optical coherence tomography (OCT) has become a mainstay in providing high-resolution, quantitative measurements in retinal evaluation. The retinal thickness measurements with different OCT imaging systems e.g., Heidelberg Spectralis and Zeiss Stratus has been well documented with established variations in measurement (1-5). Retinal thickness measurements in normal-appearing eyes additionally differ based on patient age, sex, ethnicity, and refractive error with older age, female gender, African American ethnicity, and degree of myopia (myopia of $>5 \mathrm{D}$ have thinner retinal thickness measurements) $(1,2)$.

Fundus photographs are routinely obtained (Zeiss, Topcon) in addition to OCT in evaluation and management of retinal disorders. Traditional fundus photographs (flash based) record $30-45^{\circ}$ of the retina, and require pupillary dilation. Nine-field imaging is utilized to obtain image of larger area of retina. Confocal fundus cameras based on confocal imaging technology (Optos, Clarus, RetCam, Panoret) may provide a wide field of view (up to $200^{\circ}$ ). Typically, OCT and fundus photography are performed separately. In addition to OCT, fundus photographs are routine in the documentation of retinal disease and treatment course.

The Optos Monaco Integrated Imaging System Optical Coherence Tomography (MIIS-SD OCT), a novel instrument, acquires ultra-widefield (UWF) fundus photographs $\left(200^{\circ}\right)$ as well OCT images concurrently with confocal technology and obviates the need for two separate tests. The increasing speed of data collection (A-Scan rate up to 70k cycles per second) allows enhanced resolution and better delineation of retinal layers with the assistance of built-in active eye tracking and automatic scan positioning. This unique capability of the MIIS-SD OCT reduces image acquisition time and permits improved clinic flow as both studies are completed simultaneously instead of independently. Although OCT scans may help detect morphological alterations in the retina, in the absence of normative data, it is difficult to obtain objective quantitative comparison between scans.

In this study, we sought to establish the comparative normative data for clinical use and to investigate differences in retinal thickness both in central subfoveal, as well as eight perifoveal subfields between MIIS-SD OCT and Spectralis OCT. In addition, we assessed the quality of UWF fundus photographs that were obtained in tandem in all subjects.

\section{Methods}

This is a cross-sectional study comparing retinal thickness measurements between Monaco and spectralis OCT in normal healthy subjects without known retinal disease. Study approval was obtained from the local institutional review board. The study was conducted in accordance with the Declaration of Helsinki (as revised in 2013). A written study consent was obtained as study procedures were part of routine clinical testing. Eligible study subjects were at least 18 years of age with no history of known ocular pathology, medical treatment for retinal disorders, or therapy that may have affected the structural integrity of the retina. Each subject underwent a complete ophthalmologic examination, best-corrected visual acuity (BCVA) testing utilizing the ETDRS charts, tonometry, and anterior and posterior segment examination. Exclusion criteria included history of ocular disease, intraocular pressure $>21 \mathrm{mmHg}$, history of intraocular laser or surgical treatment, BCVA $<20 / 20$, and refractive error \pm 0.50 Diopters.

Heidelberg Spectralis OCT images were obtained with the OCT camera, which centers on the subject's fovea while the operator of the OCT camera monitored stability of foveal fixation using an infrared camera. The measured scan extended from the vitreoretinal interface to the outer portion of the retinal pigmented epithelium-Bruch's membrane complex and images were obtained through dilated pupil. Retinal thickness is defined by the distance between these two interfaces at each point along the $x$-axis. Axial and transverse OCT macular scans were $7 \mu \mathrm{m}$ and $10 \mu \mathrm{m}$, respectively with production of an $8.9 \mathrm{~mm} \times 8.9 \mathrm{~mm}$ macular OCT image. Cross-sectional scans were analyzed using incorporated software with subsequent generation of mathematical values of retinal thickness.

The Optos Monaco UWF imaging device with integrated OCT was used to generate a $200^{\circ}$ singlecapture optomap fundus photograph with cross-sectional 

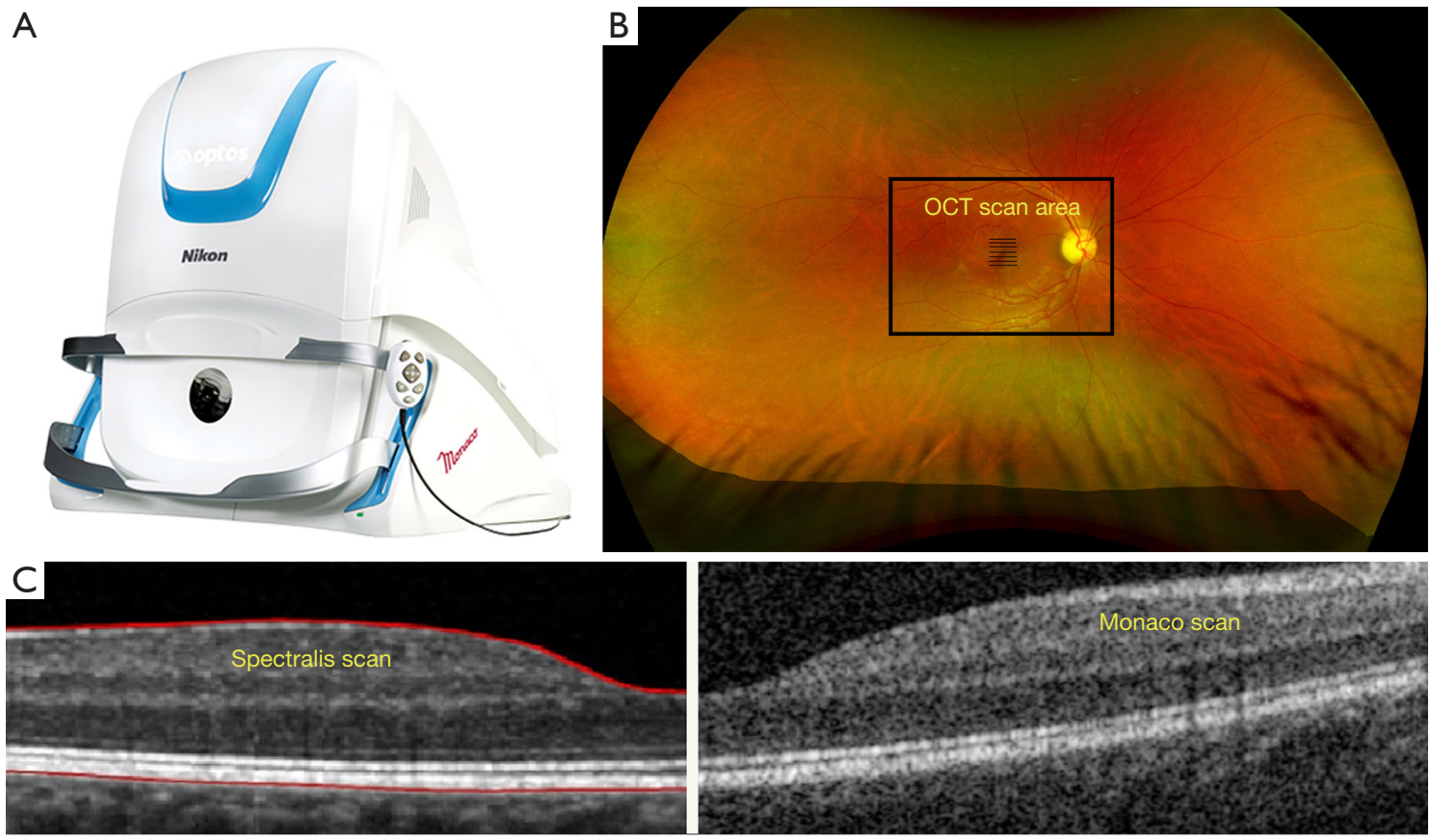

D
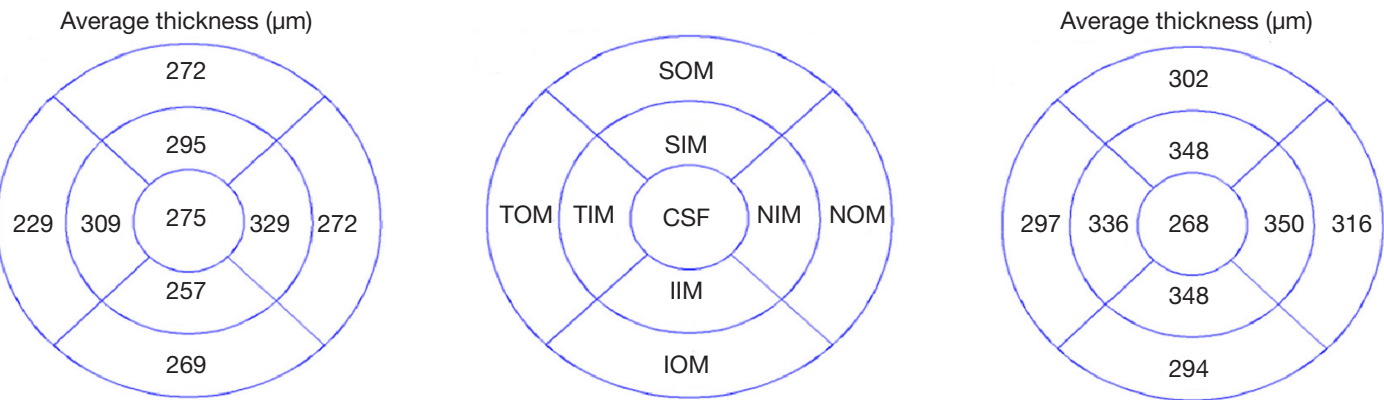

Figure 1 Optos Monaco fundus photography and optical coherence tomography (OCT) scan as compared with Heidelberg Spectralis OCT. (A) Representative image of the Optos Monaco Integrated Imaging System Optical Coherence Tomography. (B) Demonstrative fundus photo obtained by the Optos Monaco Imaging System. Up to $200^{\circ}$ of the retina are observable with the imaging device. (C) Crosssection of the macula by MIIS-SD OCT (right) and Heidelberg Spectralis OCT (left). Demonstration of retinal segmentation from anterior to posterior retinal surface in measurement of retinal thickness by MIIS-SD OCT on the right and Heidelberg Spectralis on the left. (D) Average retinal thickness obtained in subset of patients utilizing the nine ETDRS subfields on MIIS-SD OCT and Heidelberg Spectralis OCT with radii of curvature of circles 1,3 , and $6 \mathrm{~mm}$, respectively. Description of ETDRS subfields: CST, central subfield; SIM, superior inner macula; SOM, superior outer macula; NIM, nasal inner macula; NOM, nasal outer macula; IIM, inferior inner macula; IOM, inferior outer macula; TIM, temporal inner macula; TOM, temporal outer macula.

$40^{\circ}$ spectral-domain OCT scans in a single comprehensive view (Figure $1 A, 1 B$ ). The scanning laser ophthalmoscope technology combined with an ellipsoid mirror allowed for simultaneous acquisition of an optomap ultra-widefield image and OCT scan. Pupillary dilation was not required. Monaco OCT images were generated with the Monaco OCT camera, which uses a blue target as the patient looks into the device. The operator of the OCT camera monitored the correct orientation without need for focusing given built-in automatic scan-positioning. Similar to Heidelberg Spectralis OCT, the program maps the foveal umbo, and the surrounding $1.5 \mathrm{~mm}^{2}$ area extending from the interface to the outer portion of the retinal pigmented epithelium-Bruch's membrane complex.

Retinal thickness was defined by the distance between these two interfaces at each point along the $x$-axis. Each 


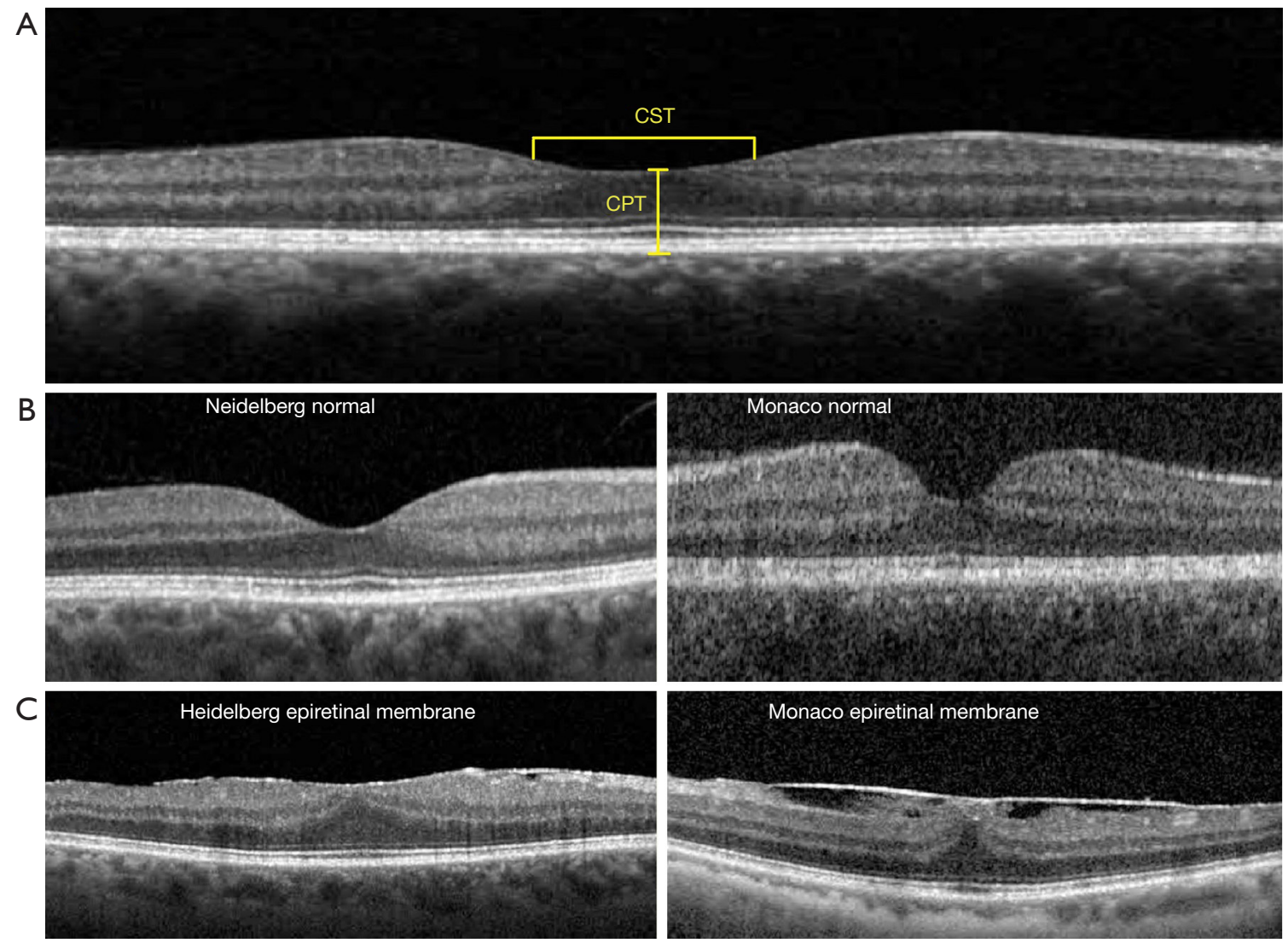

Figure 2 Optical coherence tomography (OCT) scan of the macula with demonstration of (A) Central Point Thickness (CPT) and Central Subfield Thickness (CST) measurements along with a side-by-side comparison of the Heidelberg (left) and Monaco (right) OCT scans, including both (B) normal and (C) pathological scans.

OCT image has an axial and transverse resolution of $10 \mu \mathrm{m}$ and $20 \mu \mathrm{m}$, respectively with production of a $12 \mathrm{~mm} \times 9 \mathrm{~mm}$ macular OCT image.

Image J measuring tools (National Institute of Health, Bethesda, MD, USA) was calibrated using the built-in $200 \mu \mathrm{m}$ legend of Heidelberg report. The measured scan generated by the MIIS-SD OCT spanned the same area measured by the Heidelberg OCT. Images acquired by the MIIS-SD OCT were measured using the caliper tool. We utilized the retinal map analysis protocol for both the MIIS-SD OCT and Heidelberg Spectralis OCT to average retinal thickness measurements including central subfield thickness (CST) and central point thickness (CPT) (Figure 1C,1D). CST was defined by the average of all points within the inner circular area of $1 \mathrm{~mm}$ radius centered on the umbo. CPT was defined by the direct subfoveal thickness from the inner aspect of the foveola extending to the outer portion of the retinal pigmented epithelium for all thirty-four eyes (Figure 2A,2B). The MIIS-SD OCT CPT scans were correlated to the "center" measurement in the Heidelberg Spectralis OCT report as well as the subfield foveal thickness (CST). An example of a pathological OCT scan demonstrating an epiretinal membrane is also included for comparison to our study subjects (Figure 2C). We also obtained average measurements for each of the eight subfield segments (four inner and four outer zones) as defined by the Early Treatment Diabetic Retinopathy Study (ETDRS) in a subset of our patients (10 total subjects) (6). Inner, intermediate, and outer rings with radii of 1,3 , and 6 $\mathrm{mm}$, respectively, were used for analyses (Figure 2B). Results were then analyzed using the Student's $t$-test/ANOVA test (InStat, version 3.06; GraphPad, San Diego, CA, USA) for the comparison of means and the Pearson coefficient test for the correlation studies. 
Table 1 Mean Retinal Thickness $(\mu \mathrm{m})$ and difference between averages in the ETDRS subfields by MIIS-SD OCT and Heidelberg Spectralis OCT

\begin{tabular}{lcccccccccc}
\hline Type of scan & CPT & CST & SIM & NIM & IIM & TIM & SOM & NOM & IOM & TOM \\
\hline Monaco OCT & 266 & 275 & 295 & 329 & 257 & 309 & 272 & 272 & 269 & 229 \\
Spectralis OCT & 230 & 268 & 348 & 350 & 348 & 336 & 302 & 316 & 294 & 297 \\
Difference & 36 & 7 & 53 & 21 & 91 & 27 & 30 & 44 & 25 & 68 \\
Pearson correlation coefficient $(r)$ & 0.57 & 0.59 & 0.51 & 0.52 & 0.52 & 0.60 & 0.57 & 0.51 & 0.54 & 0.52 \\
\hline
\end{tabular}

CST, central subfield; SIM, superior inner macula; SOM, superior outer macula; NIM, nasal inner macula; NOM, nasal outer macula; IIM, inferior inner macula; IOM, inferior outer macula; TIM, temporal inner macula; TOM, temporal outer macula.

\section{Results}

\section{Demographics}

Thirty-four eyes of 17 patients were included in the study with a spread amongst all races (eyes included 12 White/ Caucasian, 12 Asian, 10, Black/African American). Serial OCT scans were performed with both MIIS-SD OCT and Heidelberg Spectralis OCT imaging systems on all subjects (Figure 2B). The mean age of the participants was 40 years of age ( $\mathrm{SD} \pm 9$ years old). There was a total of 6 males and 11 females in the study population. Subjects had a negligible refractive error of \pm 0.50 Diopters without any ocular morbidity.

\section{Fundus photography}

Optos Monaco generated wide-angle fundus photographs $\left(200^{\circ}\right)$ with simultaneous spectral-domain OCT in all subjects without the need for pupillary dilation (Figure 1). The integrated imaging modality allowed for a rapid, yet wide-ranging view of the central pole, thus permitting a detailed view of the macula. Stereo images allowed for pairing for optic disc and retinal evaluation. Fundus parameters including disc, macula, vasculature and periphery were normal in all subjects.

\section{$C P T$}

The mean CPT using the MIIS-SD OCT was $268.55 \mu \mathrm{m}$ with a SD $\pm 20.70 \mu \mathrm{m}$ (for a confidence interval of $95 \%$, the standard margin of error was $268.5588 \pm 6.96( \pm 2.59 \%)$. While using the Heidelberg Spectralis OCT, the mean thickness obtained was $230.67 \mu \mathrm{m}$ with a $\mathrm{SD} \pm 17.75 \mu \mathrm{m}$ [for a confidence interval of $95 \%$, the standard margin of error was $230.6765 \pm 5.969( \pm 2.59 \%)]$. The $r$-value was 0.5697 , $\mathrm{P}<0.0004$.

\section{CST}

The mean CST using the MIIS-SD OCT was $300.53 \mu \mathrm{m}$ with a $\mathrm{SD} \pm 22.81 \mu \mathrm{m}$ [for a confidence interval of $95 \%$, the standard margin of error was $300.53 \pm 7.67( \pm 2.55 \%)]$. While using the Heidelberg OCT, the mean thickness obtained was $265.18 \mu \mathrm{m}$ with a $\mathrm{SD} \pm 17.33 \mu \mathrm{m}$ [for a confidence interval of $95 \%$, the standard margin of error was $265.18 \pm 5.83( \pm 2.20 \%)]$. The $r$-value was $0.5285, \mathrm{P}<0.0013$.

\section{ETDRS subfield analysis}

Mean retinal thickness in ETDRS subfields, as measured by MIIS-SD OCT and Heidelberg Spectralis OCT in a subset of our patients is shown in Table 1. Mean retinal thickness was highest in the nasal inner and outer fields in both MIIS and Heidelberg Spectralis OCT. The mean difference between retinal thicknesses was $44.88 \mu \mathrm{m}$ (range, 21-91 $\mu \mathrm{m}$ ) in the eight ETDRS subfields. Pearson correlation coefficient ranged from 0.51 to 0.60 . The relationship between MIIS-SD OCT and Heidelberg Spectralis OCT central subfield foveal thickness was calculated. The $r$-value was $0.53, \mathrm{P}<0.05$ (Figure 3).

\section{Discussion}

During the last decade, OCT has become a clinically useful imaging modality in providing high-resolution, quantitative data in the evaluation of macular thickness and management of retinal pathology. Previous studies have validated the reproducibility and reliability of measurements of retinal thickness (1-5). Our study is the first of its kind to demonstrate that the retinal thickness measured with MIIS-SD OCT is comparable to thickness data obtained by Spectralis OCT in healthy individuals without retinal pathology. 

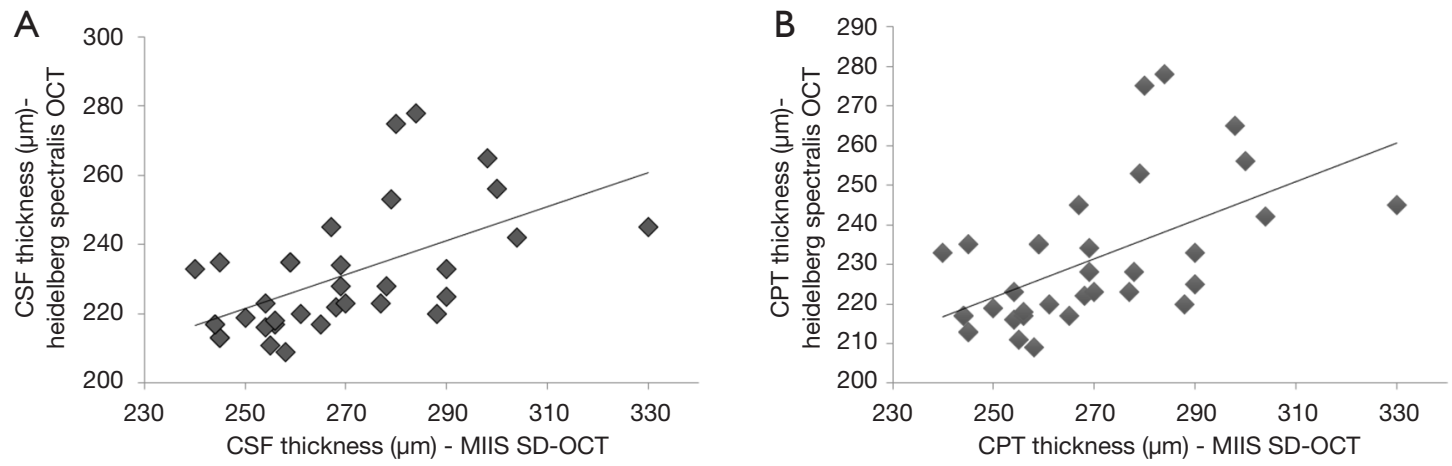

Figure 3 Correlation graph of Central Subfield Thickness (CST) and Central Point Thickness (CPT) measurements ( $\mu$ ) by MIIS-SD OCT and Heidelberg Spectralis OCT. (A) Demonstration of CST and (B) CPT utilizing both imaging modalities.

In concordance with the Heidelberg Spectralis and other OCT imaging systems, MIIS-SD OCT demonstrated similar trends in macular thickness measurements. The thinnest area of retinal thickness was indeed within the fovea, with increased parafoveal thickness, and gradual decrease in perifoveal thickness (7-9). The difference across subfield thickness (being higher in inner subfields than outer) is consistent with other high-resolution OCTs in healthy subjects (10). This normative data is consistent with the normal macular contour.

The imaging technique utilized by the MIIS-SD OCT was able to render an overall CSF that is thinner than the average described by Grover et al. (2). Measurement of retinal thickness in MIIS-SD OCT relies on definition of the anterior and posterior retinal surfaces. This variation in macular thickness measurement seen with the Monaco OCT could be due to differences in definition of the retinal boundary, which is different in various OCT instruments $(8,11)$. It is almost apparent that the cross-section displays a retinal pigment epithelium (RPE) and Bruch's membrane complex that is thicker than in the Heidelberg Spectralis imaging system. MIIS SD-OCT additionally allows for detailed mapping of the macula, whereby macular thickness is derived from more data points (70,000 axial scans/image) leading to more reproducible results (11-15).

In our study, within the 9 ETDRS subfields, retinal thickness in the $1 \mathrm{~mm}$ region was the thinnest; nasal zone of the $3 \mathrm{~mm}$ the thickest, and temporal portion of the $6 \mathrm{~mm}$ zone the thinnest. The area of the macula within $3 \mathrm{~mm}$ of the center is the thickest, and decreases towards the outer $6 \mathrm{~mm}$ region. Our results are in concordance with similar quantitative measurements performed on the Cirrus and Stratus OCTs $(10,16)$. Additionally, retinal thickness was maximal in the nasal inner quadrant with the OCT Monaco (in contrast to maximal retinal thickness in the nasal outer quadrant with Spectralis), secondary to the use of different radii of curvature in the studies (2).

Differences between Cirrus and Heidelberg Spectralis OCT have been reported and our study adds a new OCT imaging modality similar to these. Previous studies have evaluated macular thickness measurements in healthy eyes using different OCT instruments. One study found that six different OCT systems provided different results for CRT, with measurements with the Cirrus HD-OCT $(276 \pm 17 \mu \mathrm{m}$ in right eye and $277 \pm 21 \mu \mathrm{m}$ in left eye) and Heidelberg Spectralis HRA+OCT yielding the highest values (16). Other studies utilizing the Heidelberg Spectralis instrument have demonstrated mean CST thickness in the range of $270 \pm 23-24 \mu \mathrm{m}(3,7)$. In our study, the Heidelberg Spectralis OCT yielded a mean CST thickness of $265.18 \mu \mathrm{m}$ with a $\mathrm{SD} \pm 17.33 \mu \mathrm{m}$. Discrepancies seen in measuring macular thickness have been attributed to differences in retinal segmentation algorithms utilized by the various OCT devices or sample size. Cirrus HD-OCT and Heidelberg Spectralis HRA+OCT include the RPE layer in measurements, whereas other systems do not. The MIIS SD-OCT includes the RPE layer in measurements (like the Cirrus and Heidelberg Spectralis imaging systems) and yields an average thicker macular thickness $(268.55 \pm 20.39 \mu \mathrm{m})$ as demonstrated in our study. Based on the data, different OCT devices cannot be used interchangeably to measure retinal thickness. However, they are all likely to detect underlying pathology as they are based on the same optical principle.

Traditional retinal practice often entails acquisition of fundus images as well as OCT as part of clinical care. Flash 
photography (e.g., Zeiss) in conjunction with pupillary dilation typically captures $30-45^{\circ}$ of the retina. Recently non-contact, often non-mydriatic UWF imaging (Optos confocal scanning laser ophthalmoscopy, Dunfermline, UK) is used to acquire wide angle photographs. Hence two separate devices are required (fundus photography and OCT) and serial testing adds to wait time and negatively affects patient flow.

The Optos Monaco UWF device with an integrated OCT imaging system features a $200^{\circ}$ single-capture optomap fundus photograph with quick acquisition time and concurrent high-resolution OCT images. Its ease of use (built-in active eye tracking and automatic scan-positioning) enables a fast (in as little as 60 seconds to produce a singlecapture retinal image with 6-image panel including color, AF, OCT), multimodality image overview without the need for pupillary dilation, and obviates the need for separate fundus photography and OCT devices. Ultra wide angle photographs may additionally reveal peripheral retinal pathology (despite small pupils) outside the field of view of traditional small-field fundus photography and improve disease detection (2C), documentation, and management without compromising practice efficiency.

In conclusion, this study provides the first report of normative OCT data utilizing the Optos Monaco Integrated Imaging System in quantitative estimation of retinal thickness. MIIS-SD OCT retinal thickness measurements strongly correlated with and is comparable to the Heidelberg Spectralis OCT. Simultaneous highresolution $200^{\circ}$ single-capture fundus photographs is an added benefit in evaluation and management of retinal pathologies.

\section{Acknowledgments}

Funding: None.

\section{Footnote}

Conflicts of Interest: All authors have completed the ICMJE uniform disclosure form (available at https://dx.doi. org/10.21037/qims-20-1252). The authors have no conflicts of interest to declare.

Ethical Statement: The authors are accountable for all aspects of the work in ensuring that questions related to the accuracy or integrity of any part of the work are appropriately investigated and resolved. Study approval was obtained from the local institutional review board. The study was conducted in accordance with the Declaration of Helsinki (as revised in 2013). A written study consent was obtained as study procedures were part of routine clinical testing.

Open Access Statement: This is an Open Access article distributed in accordance with the Creative Commons Attribution-NonCommercial-NoDerivs 4.0 International License (CC BY-NC-ND 4.0), which permits the noncommercial replication and distribution of the article with the strict proviso that no changes or edits are made and the original work is properly cited (including links to both the formal publication through the relevant DOI and the license). See: https://creativecommons.org/licenses/by-nc-nd/4.0/.

\section{References}

1. Wagner-Schuman M, Dubis AM, Nordgren RN, Lei $Y$, Odell D, Chiao H, Weh E, Fischer W, Sulai Y, Dubra A, Carroll J. Race- and sex-related differences in retinal thickness and foveal pit morphology. Invest Ophthalmol Vis Sci 2011;52:625-34.

2. Grover S, Murthy RK, Brar VS, Chalam KV. Comparison of retinal thickness in normal eyes using Stratus and Spectralis optical coherence tomography. Invest Ophthalmol Vis Sci 2010;51:2644-7.

3. Grover S, Murthy RK, Brar VS, Chalam KV. Normative data for macular thickness by high-definition spectraldomain optical coherence tomography (spectralis). Am J Ophthalmol 2009;148:266-71.

4. Kelty PJ, Payne JF, Trivedi RH, Kelty J, Bowie EM, Burger BM. Macular thickness assessment in healthy eyes based on ethnicity using Stratus OCT optical coherence tomography. Invest Ophthalmol Vis Sci 2008;49:2668-72.

5. Wong AC, Chan CW, Hui SP. Relationship of gender, body mass index, and axial length with central retinal thickness using optical coherence tomography. Eye (Lond) 2005;19:292-7.

6. Early Treatment Diabetic Retinopathy Study design and baseline patient characteristics. ETDRS report number 7. Ophthalmology 1991;98:741-56.

7. Chalam KV, Bressler SB, Edwards AR, Berger BB, Bressler NM, Glassman AR, Grover S, Gupta SK, Nielsen JS; Diabetic Retinopathy Clinical Research Network. Retinal thickness in people with diabetes and minimal or no diabetic retinopathy: Heidelberg Spectralis optical coherence tomography. Invest Ophthalmol Vis Sci 
2012;53:8154-61.

8. Adhi M, Aziz S, Muhammad K, Adhi MI. Macular thickness by age and gender in healthy eyes using spectral domain optical coherence tomography. PLoS One 2012;7:e37638.

9. Gupta P, Sidhartha E, Tham YC, Chua DK, Liao J, Cheng CY, Aung T, Wong TY, Cheung CY. Determinants of macular thickness using spectral domain optical coherence tomography in healthy eyes: the Singapore Chinese Eye study. Invest Ophthalmol Vis Sci 2013;54:7968-76.

10. Legarreta JE, Gregori G, Punjabi OS, Knighton RW, Lalwani GA, Puliafito CA. Macular thickness measurements in normal eyes using spectral domain optical coherence tomography. Ophthalmic Surg Lasers Imaging 2008;39:S43-9.

11. Leung CK, Cheung CY, Weinreb RN, Lee G, Lin D, Pang CP, Lam DS. Comparison of macular thickness measurements between time domain and spectral domain optical coherence tomography. Invest Ophthalmol Vis Sci 2008;49:4893-7.

12. Song WK, Lee SC, Lee ES, Kim CY, Kim SS.

Cite this article as: Enghelberg M, Gasparian S, Chalam KV. Baseline retinal thickness measurements with a novel integrated imaging system (concurrent optical coherence tomography and fundus photography) positively correlates with spectralis optical coherence tomography. Quant Imaging Med Surg 2022;12(1):417-424. doi: 10.21037/qims-20-1252
Macular thickness variations with sex, age, and axial length in healthy subjects: a spectral domain-optical coherence tomography study. Invest Ophthalmol Vis Sci 2010;51:3913-8.

13. Anger EM, Unterhuber A, Hermann B, Sattmann H, Schubert C, Morgan JE, Cowey A, Ahnelt PK, Drexler $W$. Ultrahigh resolution optical coherence tomography of the monkey fovea. Identification of retinal sublayers by correlation with semithin histology sections. Exp Eye Res 2004;78:1117-25.

14. Panda-Jonas S, Jonas JB, Jakobczyk-Zmija M. Retinal photoreceptor density decreases with age. Ophthalmology 1995;102:1853-9.

15. Gao H, Hollyfield JG. Aging of the human retina. Differential loss of neurons and retinal pigment epithelial cells. Invest Ophthalmol Vis Sci 1992;33:1-17.

16. Sabouri MR, Kazemnezhad E, Hafezi V. Assessment of Macular Thickness in Healthy Eyes Using Cirrus HDOCT: A Cross-Sectional Study. Med Hypothesis Discov Innov Ophthalmol 2016;5:104-11. 